Cognitive Impairment-MCI) through a large driving simulator experiment.

Methods A full neurological and neuropsychological assessment was carried out and then a driving simulator experiment was applied. The driving tasks included driving in urban and rural road, at moderate and high traffic volumes, with and without distraction (conversation with passenger and conversation through mobile phone), while various unexpected incidents were scheduled to occur (sudden appearance of an animal on the roadway, or sudden appearance of a child chasing a ball or a car suddenly getting out of a parking position and getting in the road in urban area).

Results The sample scheme consisted of 140 participants of similar demographics: 31 healthy controls, $25 \mathrm{AD}, 59 \mathrm{MCI}$, and 25 PD patients. The accident probability was analysed, by descriptive statistics at first and then appropriate mathematical models were developed. Results indicated that patients were more likely to crash the incident that unexpectedly happened. The accident risk of $\mathrm{AD}$ and MCI drivers was 30\% higher than the control group. Finally, the negative impact of use of the mobile phone, regarding accident probability, was more significant on the patients.

Conclusions The patients had systematically higher accident probability than the cognitively intact individuals, in the majority of driving conditions. These results could have considerable practical importance as they provide useful information about the formulation of efficient countermeasures.

\section{EPIDEMIOLOGICAL SURVEILLANCE OF DROWNING: A NATIONAL SURVEY IN FRANCE, 1 JUNE TO 30 SEPTEMBER 2015}

'Linda Lasbeur, ${ }^{1}$ Emmanuelle Szego-Zguem, ${ }^{2}$ Marie-Thérèse Guillam, 'Bertrand Thélot. ${ }^{1}$ Santé Publique France, Saint-Maurice, France; ${ }^{2}$ SEPIA-Santé, Baud, France

\subsection{6/injuryprev-2016-042156.671}

Background In France, national surveys on drowning have been contributing to the epidemiological surveillance of injuries for 15 years. The purposes of these surveys were to describe the characteristics and the risk factors of unintentional drowning, and to contribute to their prevention.

Methods A prospective survey was set up in France from the 1 June to 30 September 2015. Each drowning followed by hospitalisation or death was recorded. The data were collected by fire rescue and emergency services.

Results During the 4 months, 1,217 unintentional drownings were reported (on 2015/11/23), including 435 (36\%) which resulted in death. Children under 6 represented $18 \%$ of the victims (219, including 29 deaths); adults over 45 accounted for $45 \%$ of the victims (550, including 260 deaths). The distribution by drowning place was the following: 233 in private swimming pools (68 deaths), 61 in public swimming pools (6 deaths), 156 in streams (115 deaths), 130 in lakes (65 deaths), 600 in sea water (166 deaths), and 37 in other places (15 deaths). In private swimming pools, children under 6 mainly drowned due to the lack of parental surveillance. In streams and lakes, drowning was more frequent among adults after a fall, or when engaging in solitary activities, or after having consumed alcohol. In sea water, many victims were tourists over 45 with a health problem.

Conclusions The number of drowning remained stable during the summer months since 2003, except for children under 6 in private swimming-pools, whose deaths declined from 30 in 2003 to 13 in 2015 . These results illustrate the need to strengthen prevention messages: careful surveillance of young children; learning how to swim as early as possible, since swimming is the most reported activity; not overestimating one's physical condition; swimming in supervised areas; inquiring about the state of the sea and the meteorological conditions. During the survey period, the media relayed prevention messages on TV and newspapers.

\section{ACCEPTABILITY OF CHILD DROWNING PREVENTION INTERVENTIONS IN RURAL BANGALADESH}

${ }^{1}$ Shirin Wadhwaniya, ${ }^{2}$ Kamran UI Baset, ${ }^{3}$ Shumona Sharmin Salam, ${ }^{4} \mathrm{NM}$ Rabiul Awal

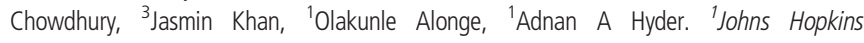
International Injury Research Unit, Department of International Health, Johns Hopkins Bloomberg School of Public Health, USA; ${ }^{2}$ Centre for Injury Prevention and Research, Bangladesh; ${ }^{3}$ International Centre for Diarrhoeal Disease Research, Bangladesh; ${ }^{4}$ Comilla University, Department of Anthropology, Bangladesh

\subsection{6/injuryprev-2016-042156.672}

Background Drowning accounts for $43 \%$ of childhood mortality among 1-4 years old in Bangladesh. The Saving of Lives from Drowning (SoLiD) project is being implemented in seven rural sub-districts of Bangladesh to assess the effectiveness of two child drowning prevention interventions - playpen and crèche. Nearly 78,000 children are receiving either playpen (plastic or wooden) or are enrolled in crèche or are receiving both. To our knowledge no study has explored the acceptability of crèche intervention for childhood drowning prevention. A previous study from Bangladesh has studied acceptability of metal playpen for childhood drowning prevention. The overall goal of this study is to explore and gain an in-depth understanding of the acceptability and perceptions of different stakeholders about crèche and playpen interventions.

Methods Qualitative research methods including 16 focus group discussions (FGDs) and 16 in-depth interviews (IDIs) will be conducted with parents of children who are receiving interventions, community-level workers who are involved in delivering these interventions and community leaders. Interview/FGD guide are developed for this. Grounded theory approach would be used for data collection and data analysis. Respondents will be recruited purposively. FGDs and IDIs will be audio-recorded, transcribed, translated, coded and thematically analysed.

Results Findings would explore stakeholder's perception and opinion about crèche and playpen; acceptability and utilisation; perceived advantages and disadvantages; challenges in using these interventions; and their relevance for child drowning prevention. Findings would also focus on stakeholder's recommendations for improving these interventions, and explore issues related to sustainability.

Conclusions This study is expected to provide inputs on the implementation and scale-up of playpen and crèche interventions for child drowning prevention in Bangladesh and other low- and middle-income countries.

\section{BANGLADESH ANCHAL AND SWIMSAFE (BASS) CHILD DROWNING PREVENTION RESEARCH- A GRAND CHALLENGE}

${ }^{1}$ Stephen Beerman, ${ }^{2}$ Mike Linnan, ${ }^{3}$ Aminur Rahman, ${ }^{3}$ Fazlur Rahman, ${ }^{4}$ Justin Scarr. ${ }^{1}$ University of British Columbia, Canada; ${ }^{2}$ The Alliance for Safe Children; ${ }^{3}$ Centre for Injury Prevention and Research, Bangladesh; ${ }^{4}$ Royal Life Saving Society Australia 